\title{
A Remodelação do Sistema Nervoso Autônomo Cardíaco pode Desempenhar um Papel na Fibrilação Atrial: Um Estudo do Sistema Nervoso Autônomo e Receptores Miocárdicos
}

\author{
Cardiac Autonomic Nervous System Remodeling May Play a Role in Atrial Fibrillation: A Study of the \\ Autonomic Nervous System and Myocardial Receptors
}

\author{
Ítalo Martins de Oliveira, ${ }^{1,2}$ (10 Evilásio Leobino da Silva Júnior, ${ }^{1,2}$ Yasmin de Oliveira Martins, ${ }^{3}$ Hermano Alexandre \\ Lima Rocha, ${ }^{4}$ Maurício Ibrahim Scanavacca, ${ }^{1}$ Paulo Sampaio Gutierrez ${ }^{10}$ \\ Instituto do Coração (InCor), Hospital das Clínicas da Faculdade de Medicina da Universidade de São Paulo, ${ }^{1}$ São Paulo, SP - Brasil \\ Hospital Messejana de Coração e Pulmão Dr. Carlos Alberto Studart Gomes, ${ }^{2}$ Fortaleza, CE - Brasil \\ Hospital Geral de Fortaleza (HGF), ${ }^{3}$ Fortaleza, CE - Brasil \\ Harvard T.H. Chan School of Public Health, ${ }^{4}$ Boston - EUA
}

\section{Resumo}

Fundamento: Alterações do substrato elétrico e anatômico do coração são fatores que originam e perpetuam a fibrilação atrial (FA), porém, os mecanismos envolvidos não foram totalmente elucidados ainda.

Objetivo: Avaliar o papel do remodelamento do sistema nervoso cardíaco intrínseco (SNCI), incluindo fibras nervosas e receptores muscarínicos e $\beta$-adrenérgicos, na FA permanente humana.

Métodos: Foram avaliadas 4 amostras em átrios de 13 corações obtidos em necrópsias de pacientes com doença cardíaca e FA permanente, e em 13 controles com as mesmas doenças, porém, sem FA. Utilizando imunoperoxidase e histomorfometria, quantificamos a densidade das fibras do $\mathrm{SNCl}$, bem como a porcentagem positiva de miocárdio para receptores $\beta$-adrenérgicos 1, 2 e 3, receptor quinase 5 acoplado à proteína $G$ (GRK-5), e receptores muscarínicos 1 a 5. Os resultados foram comparados usando ANOVA e ANOVA hierarquizada e ajustados pelo volume do átrio esquerdo e, para avaliação da expressão de receptores $\beta$ e GRK-5, pelo uso de $\beta$-bloqueadores. Adotamos como significativo $\alpha=0,05$.

Resultados: Houve aumento na densidade das fibras $(p<0,01)$, especialmente nas fibras simpáticas $(p=0,02)$. Quanto aos receptores muscarínicos, só houve diferença nos $M 1$, que estavam aumentados $(5,87 \pm 4,52$ vs $2,85 \pm 2,40 ; p=0,03)$. Quanto aos componentes do sistema adrenérgicos analisados, houve expressão aumentada de $\beta$-3 (37,41 vs 34,18, $p=0,04)$ e GRK-5 $(51,16$ vs 47,$66 ; p<0,01)$. O uso de $\beta$-bloqueadores não teve impacto na expressão de receptores beta.

Conclusão: $\mathrm{O}$ aumento na inervação do $\mathrm{SNCI}$ e a alteração na expressão de receptores em regiões suscetíveis de desencadear FA podem ter um papel na fibrilação atrial permanente.

Palavras-chave: Fibrilação Atrial/fisiopatologia; Sistema Nervoso Autônomo; Neurotransmissores; Miocárdio.

\footnotetext{
Abstract

Background: The primary factors that originate and perpetuate atrial fibrillation (AF) are electrical and anatomical substrate alterations. However, the central mechanisms governing AF perpetuation have not been elucidated yet, which is reflected on the modest results of the treatment in patients with long persistent AF.

Objective: To evaluate if human intrinsic cardiac autonomic nervous system (ICANS) remodeling, including nervous system fibers and muscarinic and $\beta$-adrenergic receptors, play a role in permanent $A F$.

Methods: Heart necropsy samples from thirteen patients with heart disease and permanent AF and thirteen controls without AF were used. By using immunoperoxidase and histomorphometry quantification, we identified the following: the density of all fibers of the ICANS, sympathetic and parasympathetic fibers; and the percentage of myocardium positive for $\beta$-adrenergic receptors 1, 2 and 3; G protein-coupled receptor kinase-5 (GRK-5); and muscarinic receptors M1 to M5. The results were compared using ANOVA and nested ANOVA and were adjusted
}

Correspondência: Paulo Sampaio Gutierrez •

Laboratório de Anatomia Patológica - Instituto do Coração, Hospital das Clínicas FMUSP - Av. Enéas Carvalho Aguiar, 44. CEP 05403-000, São Paulo, SP - Brasil E-mail: paulo.gutierrez@incor.usp.br

Artigo recebido em 29/06/2020, revisado em 29/09/2020, aceito em 25/11/2020

DOI: https://doi.org/10.36660/abc.20200725 
according to the left atrium volume for all variables, and $\beta$-blocker use to evaluate the expression of $\beta$-receptors and GRK-5.

Results: There was an overall increase in the density of fibers of the ICANS ( $p=0.006)$, especially in atrial sympathetic nerve fibers ( $p=0.017)$. Only M1 muscarinic receptors were increased (5.87 vs 2.35, $p=0.032$ ). For adrenergic receptors, the results were positive for increased expression of $\beta-3$ (37.41 vs 34.18, $p=0.039)$ and GRK-5 (51.16 vs 47.66; $p<0.001)$. $\beta$-blocker use had no impact on $\beta$-receptor expression.

Conclusion: Increased ICANS innervation and remodeling receptor expression in regions prone to triggering AF may play a role in permanent AF. Keywords: Atrial Fibrillation/physiopathology; Autonomic Nervous System; Neurotransmitter Agents; Myocardium.

Full texts in English - http://www.arquivosonline.com.br

\section{Introdução}

Alterações nos substratos elétrico e anatômico do coração constituem o fator primário que origina e perpetua a fibrilação atrial (FA). Em pacientes com FA sem doença estrutural do coração, focos ectópicos originados nas veias pulmonares têm papel bem definido como desencadeante de FA paroxística. ${ }^{1}$ No entanto, a FA é, na maior parte dos casos, secundária a doenças cardíacas estruturais, como doença isquêmica do coração, doenças valvares e outras, que apresentam consequências hemodinâmicas e anatômicas, tais como aumento do átrio esquerdo, que estão associadas à progressão da arritmia. ${ }^{1}$

A fibrose é também amplamente vista como fator independente relacionado à FA persistente em corações com alterações estruturais. ${ }^{2}$ Ela, porém, não explica totalmente a arritmia, sendo mais associada às doenças subjacentes do que à FA persistente em si. $^{3}$

A avaliação eletrofisiológica demonstrou não só uma efetiva heterogeneidade do período refratário, mas também a anisotropia das propriedades de condução, tanto nas veias pulmonares quanto nos seus óstios atriais, o que pode causar a reentrada de estímulos elétricos. ${ }^{4}$ Entretanto, os mecanismos cruciais que governam a perpetuação da FA não foram elucidados por completo - o que se reflete em resultados modestos no tratamento de pacientes com FA persistente prolongada. ${ }^{5}$

Estudos básicos e clínicos sugeriram haver participação significativa do sistema nervoso autônomo cardíaco intrínseco (SNACI) no desencadeamento e na manutenção da FA. ${ }^{6,7} \mathrm{~A}$ ativação do $\mathrm{SNACl}$ pode causar mudanças importantes no período refratário atrial, inclusive aumento na dispersão da refratariedade, que é um dos importantes mecanismos de desenvolvimento da FA persistente. ${ }^{1,8-10}$

Estudos experimentais mostraram hiperinervação simpática em cães com FA, e aumento na inervação simpática e parassimpática em áreas relacionadas a essa arritmia em animais com insuficiência cardíaca. ${ }^{11}$ Uma relação entre o SNACI e a FA foi também relatada em seres humanos, porém, em comparação com pacientes saudáveis. ${ }^{2,12}$ A possibilidade de envolvimento de distúrbios em fibras e receptores do SNACI na fibrilação atrial humana foi pouco explorada.

Assim, o objetivo do presente estudo foi avaliar o sistema nervoso autônomo cardíaco intrínseco, incluindo fibras simpáticas e parassimpáticas, e a expressão atrial de cinco tipos de receptores muscarínicos e três adrenérgicos, assim como do receptor quinase 5 acoplado à proteína G (a qual controla a expressão dos receptores adrenérgicos). Estudamos corações de pacientes com doença estrutural e fibrilação atrial permanente e, como controles (o que foi um fator importante), os de pacientes portadores das mesmas doenças, mas sem fibrilação atrial.

\section{Métodos}

Este estudo foi guiado pelos princípios da Declaração de Helsinque e aprovado pela Comissão Científica do Instituto do Coração (InCor) do Hospital das Clínicas, da Faculdade de Medicina da Universidade de São Paulo, São Paulo, Brasil (\#SDC 3043/07/118).

\section{Pacientes}

Foram utilizadas partes das amostras de estudo anterior. ${ }^{3}$ Analisamos 13 corações de adultos (acima dos 18 anos) com FA permanente registrada em prontuário com duração mínima de 2 anos, ${ }^{1}$ que foram submetidos a necrópsia (realizada em até menos de 24 horas após a morte) no Laboratório de Anatomia Patológica do InCor. Todos os pacientes tinham doenças subjacentes: doença isquêmica do coração (4), valvopatias (4), miocardiopatia hipertensiva (2), miocardiopatia dilatada idiopática (2) ou doença de Chagas, forma crônica cardíaca (1). Para evitar fatores de interferência ligados às doenças de base, corações de 13 pacientes submetidos a necrópsia no mesmo laboratório foram selecionados como controles, com pareamento de acordo com as doenças dos que tinham fibrilação atrial, mas sem qualquer referência a essa arritmia em seus prontuários. Em ambos os grupos, pacientes que tivessem sido submetidos a cirurgia ou a qualquer procedimento com potencial de modificar a estrutura cardíaca ou com cardiopatias congênitas foram excluídos.

\section{Amostras dos corações}

De cada coração foram obtidas quatro amostras, contendo epicárdio, miocárdio e endocárdio: na parede posterior do átrio direito (Figura 1A); na junção da veia pulmonar superior esquerda com o átrio esquerdo (Figura 1B); na porção medial do trajeto da veia de Marshall (Figura 1C); e em torno do coxim gorduroso superior esquerdo (os coxins gordurosos são concentrações de gordura epicárdica nas quais há tecido nervoso, sendo conhecidos como fat-pads). Tais áreas foram escolhidas porque foram antes implicadas na fibrilação atrial, e são comumente analisadas em outros estudos, ${ }^{3,12,13}$ com 


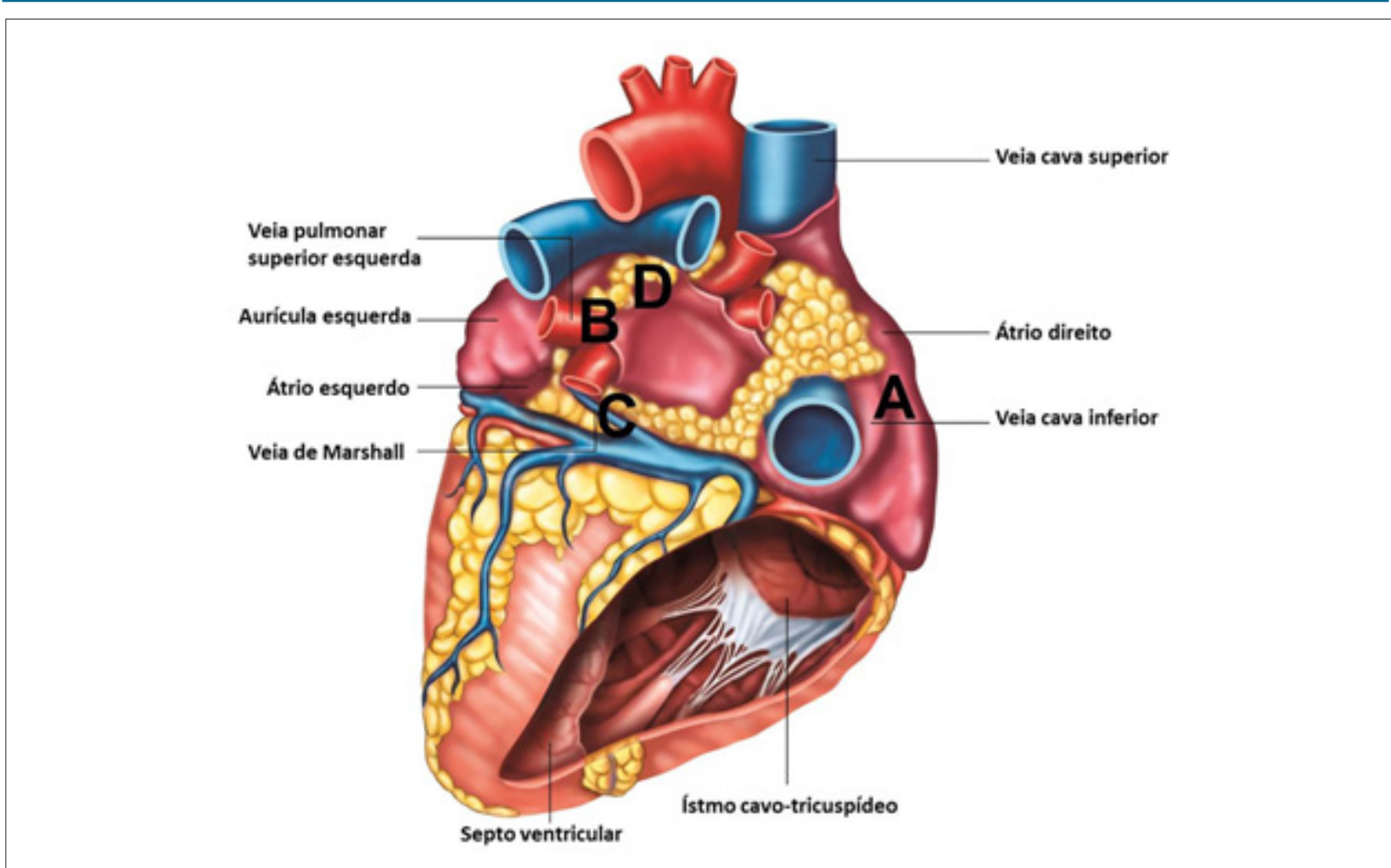

Figura 1 - Imagem fotorrealística de vista posterior do coração humano. Quatro amostras foram coletadas nas seguintes localizações: A) parede posterior do átrio direito. B) junção da veia pulmonar superior esquerda com o átrio esquerdo; C) Segmento medial da rota da veia de Marshall; D) coxim gorduroso superior esquerdo.

exceção da parede posterior do átrio direito, selecionada para verificar se eventuais alterações seriam difusas nos átrios. As localizações são apresentadas na figura 1.

Após processamento histológico convencional e embebição em parafina, cortes dessas amostras com 4 micrômetros de espessura foram preparados para a quantificação da inervação autonômica, receptores adrenérgicos e muscarínicos e expressão de GRK-5.

\section{Quantificação dos receptores e das fibras nervosas autonômicas}

A positividade forte para receptores adrenérgicos e muscarínicos, GRK-5 e área total de miocárdio considerada foram medidas por detecção automática de cor em 3 campos microscópicos em cada lâmina. Para evitar viés de seleção na escolha dos campos, foram analisados os mais distantes da etiqueta das lâminas.

As fibras nervosas autonômicas também foram quantificadas nas amostras. A proteína S-100 tem positividade em todos os nervos, enquanto a tirosina hidroxilase $(\mathrm{TH})$ marca apenas as fibras adrenérgicas (simpáticas) pós-ganglionares. Assim como outros autores, ${ }^{14}$ avaliamos custo-efetividade e consideramos os nervos TH-positivos como pertencendo ao sistema nervoso simpático; já os parassimpáticos corresponderam aos positivos para S-100 e negativos para TH. Diferentemente do que o empregado com os receptores, os cortes histológicos foram analisados por inteiro, e suas áreas e números de nervos foram quantificados em cada lâmina. Foram, então, calculadas as seguintes variáveis: porcentagem de área positiva (área positiva/ área do corte), densidade média de nervos positivos (número de nevos positivos/ área do corte) e área média dos nervos (área positiva/ número de nervos). Calculamos também o número total de fibras nervosas (S-100 positivas), fibras nervosas simpáticas (TH-positivas) e fibras nervosas parassimpáticas (S100-positivas e TH-negativas, diferença entre o total de fibras e as simpáticas).

Para aumentar o contraste entre positividade fraca e forte, as diluições para os receptores e o GRK-5 foram supraótimas ${ }^{15}$ quando comparadas ao padronizado em outros tecidos. Como controle das reações, o anticorpo primário foi omitido em 5 lâminas escolhidas ao acaso. Os cortes foram examinados em sistema de análise de imagens Axiovision 4.6, acoplado ao microscópio Axion imager A1 (ambos da Carl Zeiss, Alemanha) por observador que desconhecia a que grupo pertenciam as lâminas.

Especificação e diluição dos anticorpos: receptor muscarínico 1 (AB5164)- 1:100; receptor muscarínico 2 (AB9452)- 1:800; receptor muscarínico 3 (AB9451)1:200; receptor muscarínico 4 (AB9219)- 1:400; receptor muscarínico 5 (AB9453)- 1:400; receptor adrenérgico $\beta 1$ (SC568) - 1:200; receptor adrenérgico $\beta 2$ (SC570) - 1:50; receptor adrenérgico $\beta 3$ (SC1473) - 1:20; quinase GRK-5 (SC 565) - 1:200; S-100 (Z0311) - 1:300; tirosina hidroxilase (MAB318) - 1:50. 
O anticorpo para S-100 foi fornecido por Dako, Dinamarca; os anticorpos para tirosina hidroxilase e receptores muscarínicos, por Chemicon, Estados Unidos da América; e os anticorpos para receptores adrenérgicos e GRK-5, por Santa Cruz Biotechnology, Estados Unidos da América.

\section{Análises estatísticas}

Inicialmente, as frequências absolutas e relativas foram calculadas para as variáveis categóricas, e as medidas de tendência central e dispersão para as numéricas. Para comparar casos com controles foram utilizados os testes de qui-quadrado e t de Student. Os testes paramétricos foram usados após o teste de Kolmogorov-Smirnov para avaliação da normalidade em todas as variáveis, e estimativas de erros robustos foram usadas em modelos regressivos. A análise de variância (ANOVA) one-way foi aplicada considerando-se cada conjunto de amostras iguais para identificar as diferenças entre elas. A análise de covariância foi também realizada para ajuste das dimensões do átrio e uso de $\beta$-bloqueadores, quando apropriado, ao analisar os cortes individuais. Modelos lineares gerais (também conhecidos como análise de variância hierarquizada [nested ANOVA]) de todas as amostras histológicas dos participantes individuais) foram também aplicados para identificar o impacto do determinante principal (a saber, tratamento, um fator intersujeitos) nas diferentes variáveis dependentes. Finalmente, múltiplos modelos hierarquizados lineares gerais foram aplicados a todos os cortes histológicos para cada caso. Consideramos significantes valores de $p$ iguais a ou menores que 0,05. Em todos os modelos, foram realizados ajustes de Bonferroni nos valores de p. As análises foram efetuadas no programa SPSS, versão 23 (IBM, Inc, Estados Unidos da América).

Assim como em nosso estudo prévio de aspectos histológicos, inclusive fibrose, já que o volume atrial difere entre pacientes com e sem fibrilação atrial, fizemos a análise de sensibilidade com métodos de ajuste considerando as diferenças no tamanho do átrio esquerdo. A seguir, estimamos os resultados de cada variável em corações de qualquer grupo com um tamanho específico de átrio esquerdo para verificar se potenciais diferenças entre grupos poderiam estar ligadas a essa covariável. Adicionalmente, o uso de $\beta$-bloqueadores foi incluído para ajuste da avaliação de receptores $\beta$-adrenérgicos e de GRK-5.

\section{Resultados}

As características clínicas, morfológicas e ecocardiográficas dos pacientes com fibrilação atrial permanente e seus controles são mostradas na tabela 1 .

Dados relativos às fibras nervosas, considerando cada território amostrado, bem como todos em conjunto, são apresentados na tabela 2. Levando-se em consideração separadamente cada localização, não são observadas diferenças quanto à densidade de fibras autonômicas intrínsecas. A análise englobando todas as amostras demonstra aumento de nervos simpáticos nos pacientes com FA $\left(8,53 \pm 20,25 / \mathrm{cm}^{2}\right.$ vs $\left.2,67 \pm 4,57 / \mathrm{cm}^{2}, \mathrm{p}=0,04\right)$. Após ajuste quanto ao tamanho do átrio esquerdo, aparece um aumento

Tabela 1 - Dados clínicos e ecocardiográficos de pacientes com FA permanente e controles

\begin{tabular}{|c|c|c|c|}
\hline Variáveis & Casos com FAp $(n=13)$ & Controles $(n=13)$ & p \\
\hline Pacientes do sexo masculino [n/(\%)] & $5(38,5)$ & $8(61,5)$ & 0,24 \\
\hline Idade (anos) [média/(dp)] & $67,5(15,4)$ & $65,5(11,4)$ & 0,71 \\
\hline \multicolumn{4}{|l|}{ Doença cardíaca subjacente [n/(\%)] } \\
\hline Doença isquêmica do coração & $4(30,8)$ & $4(30,8)$ & \\
\hline Doença da válvula, incluindo DR & $4(30,8)$ & $4(30,8)$ & \\
\hline Cardiopatia hipertensiva & $2(15,4)$ & $2(15,4)$ & \\
\hline Cardiomiopatia dilatada idiopática & $2(15,4)$ & $2(15,4)$ & \\
\hline Doença de Chagas & $1(7,7)$ & $1(7,7)$ & \\
\hline Peso (kg) [média/(dp)] & $66,5(14,1)$ & $63,8(15,0)$ & 0,67 \\
\hline Altura (cm) [média/(dp)] & $162,4(14,7)$ & $160,8(8,8)$ & 0,78 \\
\hline IMC $\left(\mathrm{kg} / \mathrm{m}^{2}\right)$ [média/(dp)] & $25,0(2,9)$ & $24,5(4,2)$ & 0,74 \\
\hline Diabetes mellitus $[\mathrm{n} /(\%)]^{*}$ & $3(23,1)$ & $3(25,0)(n=12)$ & 0,99 \\
\hline Uso de beta-bloqueadores & $5(38,4)$ & $5(38,4)$ & \\
\hline Hipertensão arterial sistêmica - [n/(\%)]* & $9(69,2)$ & $4(33,3)(n=12)$ & 0,07 \\
\hline Volume de átrio esquerdo no eco [média/(dp] & $83,2(38,4)$ & $47,9(40,8)$ & 0,03 \\
\hline Espessura do septo do VE [média/(dp)] & $10,3(2,4)$ & $10,4(1,6)$ & 0,94 \\
\hline Fração de ejeção do VE [média/(dp)] & $49,8(20,1)$ & $46,1(19,8)$ & 0,67 \\
\hline Razão colágeno/colágeno+miocárdio [média +(dp)] & $0,26(0,09)$ & $0,23(0,06)$ & 0,35 \\
\hline
\end{tabular}

FAp: fibrilação atrial permanente; $n$ : número de casos; dp: desvio padrão; DR: doença reumática; IMC: índice de massa corporal; * sem informação sobre um paciente controle; eco - ecocardiograma; VE: ventrículo esquerdo. Adaptado de Oliveira IM et al.3 


\begin{tabular}{|c|c|c|c|c|c|c|}
\hline \multirow{2}{*}{$\begin{array}{l}\text { Fibras } \\
\text { Grupo }\end{array}$} & \multicolumn{2}{|c|}{$\begin{array}{l}\text { Todos }(\mathrm{S} 100) \\
\text { (unidades/cm²) }\end{array}$} & \multicolumn{2}{|c|}{$\begin{array}{l}\text { Nervo simpático }(\mathrm{TH}+) \\
\left.\quad \text { (unidades } / \mathrm{cm}^{2}\right)\end{array}$} & \multicolumn{2}{|c|}{$\begin{array}{l}\text { Nervo parassimpático } \\
\left.\text { (TH-) (unidades } / \mathrm{cm}^{2}\right)\end{array}$} \\
\hline & FAp & Controle & FAp & Controle & FAp & Controle \\
\hline \multirow{2}{*}{$A D$ - parede posterior } & $8,85 \pm 9,40$ & $9,10 \pm 5,15$ & $0,37 \pm 0,99$ & $0,50 \pm 1,14$ & $8,48 \pm 9,57$ & $8,59 \pm 5,07$ \\
\hline & p 0,935, & $0,710^{*}$ & p 0,753, & $0,905^{*}$ & p 0,971, & $0,700^{*}$ \\
\hline \multirow{2}{*}{$\begin{array}{l}\text { AE - junção da veia pulmonar } \\
\text { superior esquerda }\end{array}$} & $41,61 \pm 35,79$ & $25,78 \pm 20,90$ & $19,74 \pm 34,26$ & $4,95 \pm 6,78$ & $21,86 \pm 14,78$ & $20,83 \pm 20,47$ \\
\hline & p 0,181, & $0,256^{*}$ & p 0,140, & $0,158^{*}$ & p 0,884, & $0,918^{*}$ \\
\hline \multirow{2}{*}{$\begin{array}{l}\text { AE - meio da rota da veia de } \\
\text { Marshall }\end{array}$} & $40,15 \pm 60,28$ & $14,90 \pm 9,48$ & $5,58 \pm 9,56$ & $2,39 \pm 4,76$ & $34,56 \pm 58,07$ & $12,51 \pm 9,48$ \\
\hline & p 0,149, & $0,390^{*}$ & p 0,292, & $0,230^{*}$ & p 0,189, & $0,500^{*}$ \\
\hline \multirow{2}{*}{ FP - superior à esquerda } & $38,05 \pm 55,72$ & $19,25 \pm 11,95$ & $8,42 \pm 16,07$ & $2,85 \pm 2,82$ & $29,62 \pm 40,56$ & $17,47 \pm 10,53$ \\
\hline & p 0,246, & $0,637^{*}$ & p 0,248, & $0,666^{*}$ & p 0,325, & $0,681^{*}$ \\
\hline \multirow{2}{*}{ Amostras em conjunto } & $32,16 \pm 45,76$ & $17,26 \pm 14,20$ & $8,53 \pm 20,25$ & $2,67 \pm 4,57$ & $23,63 \pm 36,77$ & $14,80 \pm 13,27$ \\
\hline & p 0,136e, & $0,001^{\mp}$ & p 0,044 & $0,017^{\top}$ & p $0,237^{e}$ & $0,001^{\Phi}$ \\
\hline
\end{tabular}

Dados apresentados como média \pm desvio padrão. FAp: fibrilação atrial permanente; $A D$ : átrio direito; $A E$ : átrio esquerdo; FP: coxim gorduroso ("fat pad"). $p$ valor ANOVA não ajustado, ${ }^{*}$ ANOVA ajustado pelo tamanho de átrio esquerdo; ${ }^{e}$ ANOVA hierarquizado não ajustado; ${ }^{\mathrm{e}}$ ANOVA hierarquizado ajustado pelo tamanho de átrio esquerdo.

também nos nervos parassimpáticos e na quantidade total de nervos. A figura 2 (A e B) mostra a imunoexpressão das fibras nervosas em nossas amostras.

Os resultados da expressão de receptores muscarínicos e adrenérgicos e de GRK-5 são apresentados na tabela 3. Estão divididos conforme a localização das amostras, havendo ainda dados da reunião de todas elas.

A imunomarcação de receptores muscarínicos é mostrada na figura 2, C e D. Não existe diferença considerável entre as regiões subepicárdica e subendocárdica. Em corações de pacientes com FA permanente, a expressão de todos os tipos de receptores muscarínicos, com exceção do 5, estava aumentada em ao menos um território. Houve mais alterações no coxim gorduroso superior esquerdo e na veia oblíqua do átrio esquerdo (veia de Marshall). No entanto, após ajuste quanto ao tamanho do átrio esquerdo, apenas a expressão de M1 no átrio direito (e, consequentemente, a avaliação global) e M2 junto ao coxim gorduroso permaneceram significantes.

Em relação aos receptores $\beta$-adrenérgicos e GRK-5, não foi encontrada diferença na análise global dos subtipos 1 e 2 (apenas aumento em uma amostra de cada). Porém, $\beta-3$ e GRK-5 apresentaram aumento em todas as localizações na análise ajustada. Não foi detectada diferença entre pacientes que tomavam $\beta$-bloqueadores e os que não o faziam (dados não apresentados).

\section{Discussão}

\section{O sistema nervoso autônomo cardíaco intrínseco na fibrilação atrial permanente}

O SNACI corresponde a uma rede neural composta por fibras nervosas e plexos ganglionares (simpáticos e parassimpáticos) encontrados no coração e nas grandes veias adjacentes. ${ }^{16}$ Tem papel importante na fisiopatologia da FA, como demonstrado por estimulação elétrica ou por injeções de parassimpatomiméticos. ${ }^{17}$ Os dados atuais indicam não somente uma função importante na ativação dos eixos simpático e parassimpático, mas também que a modificação do balanço entre suas ações está envolvida na iniciação da FA. 8,18

Neste estudo, realizamos análise abrangente do SNACI, enfocando tanto nos nervos quanto nos receptores muscarínicos e beta-adrenérgicos. Observamos aumento de fibras nervosas autonômicas atriais, em particular dos nervos simpáticos. Entretanto, ao se analisar cada localização isoladamente, tais diferenças não se mantêm. Por outro lado, quando se faz ajuste considerando o volume do átrio esquerdo, os resultados permanecem os mesmos. Esses últimos dados sugerem que há alteração significativa na densidade de nervos em pacientes com FA permanente, ainda que levando em conta o aumento do átrio esquerdo.

Diversos artigos ${ }^{12,14,19,20}$ relataram inervação autonômica aumentada em áreas eletrofisiologicamente relacionadas à FA, tais como as veias pulmonares, os seios coronários e a veia de Marshall. Esses estudos compararam apenas a densidade de nervos (simpáticos ou parassimpáticos) nessas regiões, ou em outras, com o plexo ganglionar no miocárdio atrial. No entanto, esses territórios próximos aos plexos ganglionares têm grande densidade de nervos, mas que não obrigatoriamente estão vinculados à FA. Nossos dados revelam grande concentração do $\mathrm{SNACI}$ nessas áreas, especialmente inervação simpática. A densidade aumentada de nervos simpáticos pode ser um potencial desencadeante de arritmia causada pela inervação próxima ao plexo ganglionar e à resultante ativação do sistema nervoso autônomo, como já demonstrado em estudos experimentais. ${ }^{12-20}$

\section{Receptores muscarínicos na fibrilação atrial permanente}

A estimulação dos neurônios parassimpáticos pósganglionares libera acetilcolina (mediador colinérgico), a qual atua nos receptores muscarínicos na membrana celular em órgãos-alvo (no caso do coração, na membrana 


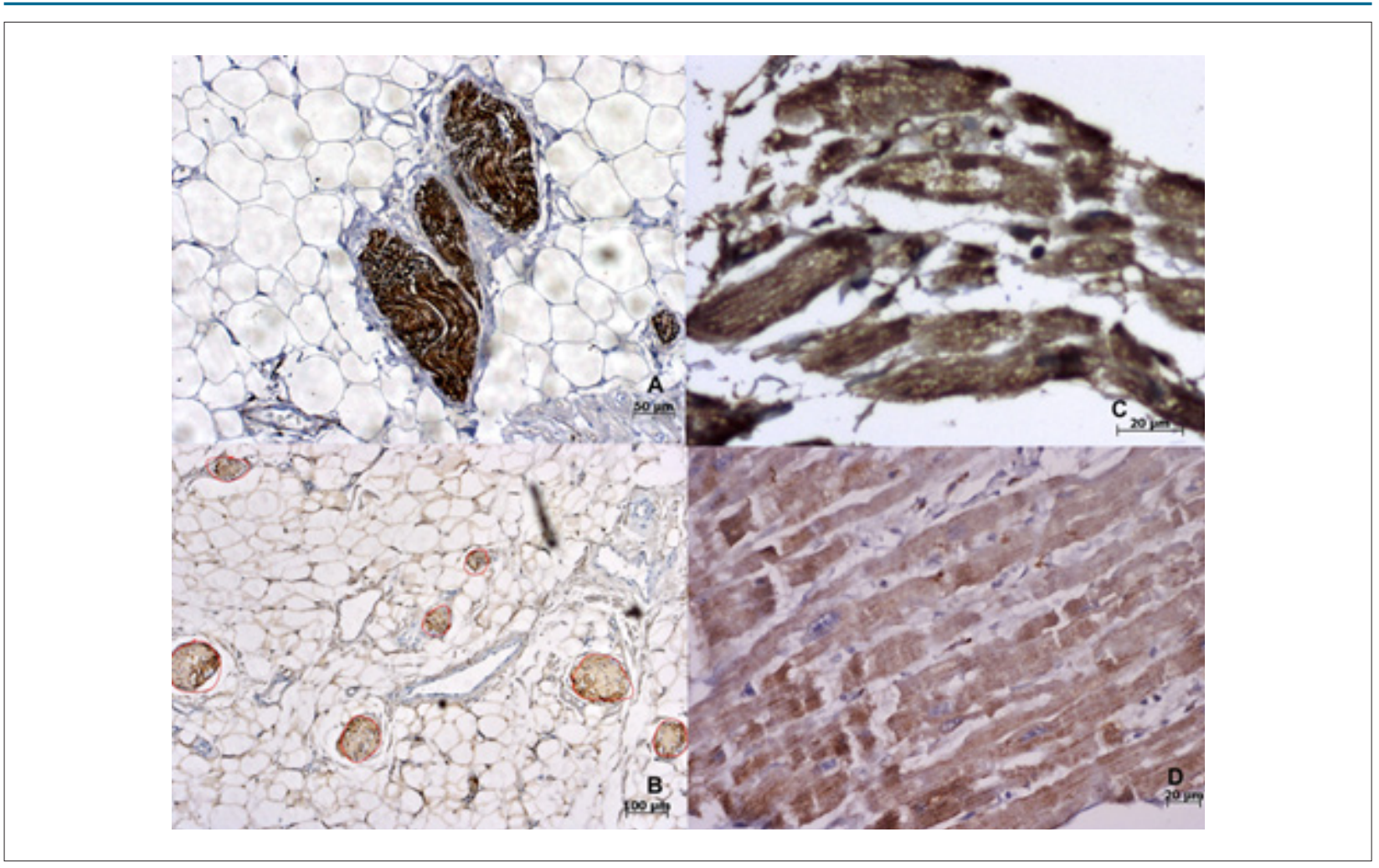

Figura 2 - A) Fibras nervosas fortemente positivas para tirosina-hidroxilase, portanto consideradas fibras simpáticas; B) Fotomicrografia da tela do sistema de análise de imagens mostrando nervos marcados para proteína S-100; C e D) Áreas positivas (C) e negativas (D) em cortes histológicos de miocárdio com reação imuno-histoquímica para o receptor muscarínico 1.

dos miócitos). ${ }^{21}$ Foram descritos cinco tipos de receptores muscarínicos (M1 a M5), cuja presença em seres humanos foi demonstrada por Wang et al., ${ }^{22}$ em estudo descritivo de amostras de átrios direitos obtidas de 4 pacientes submetidos à cirurgia de revascularização do miocárdio. ${ }^{22}$ No presente trabalho, a expressão de todos esses receptores (exceto M5) estava aumentada em corações de portadores de FA em comparação à dos controles. A mais significativamente alterada foi a do receptor M1, inclusive nas análises ajustadas, como apresentado na tabela 3. Todas as localizações exibiam aumento significativo desse receptor, com exceção da junção da veia pulmonar superior esquerda. O aumento de M1 no miocárdio de pessoas com FA permanente pode estar diretamente relacionado com a fibrilação em si, e ajuda a explicar o aumento anteriormente descrito do tônus simpático por liberação de catecolaminas nos terminais nervosos simpáticos, com efeito estimulador induzido por estas. ${ }^{23}$

Os receptores 2, 3 e 4 estavam aumentados nos pacientes com FA em apenas um local: 2 e 3 próximos ao coxim gorduroso superior esquerdo, e 4 na região da veia de Marshall. Além dos receptores M1 e M2, o M4 foi encontrado nos gânglios simpáticos e pode ser induzido por catecolaminas, de forma similar ao receptor M1. De acordo com estudo de Makino et al., a região da veia de Marshall tem grande número de fibras nervosas simpáticas e de gânglios parassimpáticos, e pode de fato ter um papel ligado à expressão aumentada desses receptores. ${ }^{14}$ Assim, as áreas afetadas são de fato as mais relacionadas à $\mathrm{FA}$; apenas o M1 parece ter alteração mais difusa, atingindo o átrio direito e o esquerdo.

Foram descritas alterações na expressão de receptores muscarínicos em modelos experimentais, o que pode sugerir que eles tenham uma função na fisiopatologia, e talvez no tratamento, da FA. Em modelo experimental de insuficiência cardíaca em cães, as densidades dos receptores M2 e M4 estavam reduzidas, e as dos receptores, aumentadas nos átrios com FA, em comparação com os sem FA. ${ }^{24}$ Deve-se salientar que M2 e M4 inibem os canais de cálcio, e o M2 tem ações inotrópicas e cronotrópicas. ${ }^{21,22}$ Assim, seria possível esperar que esses receptores estivessem diminuídos, e não aumentados, na FA permanente. O mesmo não se aplica aos receptores $\mathrm{M} 1$ e $\mathrm{M} 3$, de quem foram documentadas funções estimuladoras em outros órgãos. ${ }^{22} \mathrm{O}$ receptor M5 e suas ações são pouco conhecidos nos corações humanos, mas de todo modo quanto a ele não houve diferença entre os grupos.

Nossos resultados sugerem que o tecido miocárdico adjacente aos plexos ganglionares pode estar associado à expressão aumentada de receptores muscarínicos, exceto no caso do M5. A expressão aumentada do receptor muscarínico ocorreu mais frequentemente na porção do átrio esquerdo, onde se situa a veia de Marshall.

Embora não tenhamos avaliado função, algumas considerações sobre a fisiopatologia da FA permanente podem ser feitas com base em nossas observações morfológicas. Primeiramente, é necessário considerar a possibilidade de 


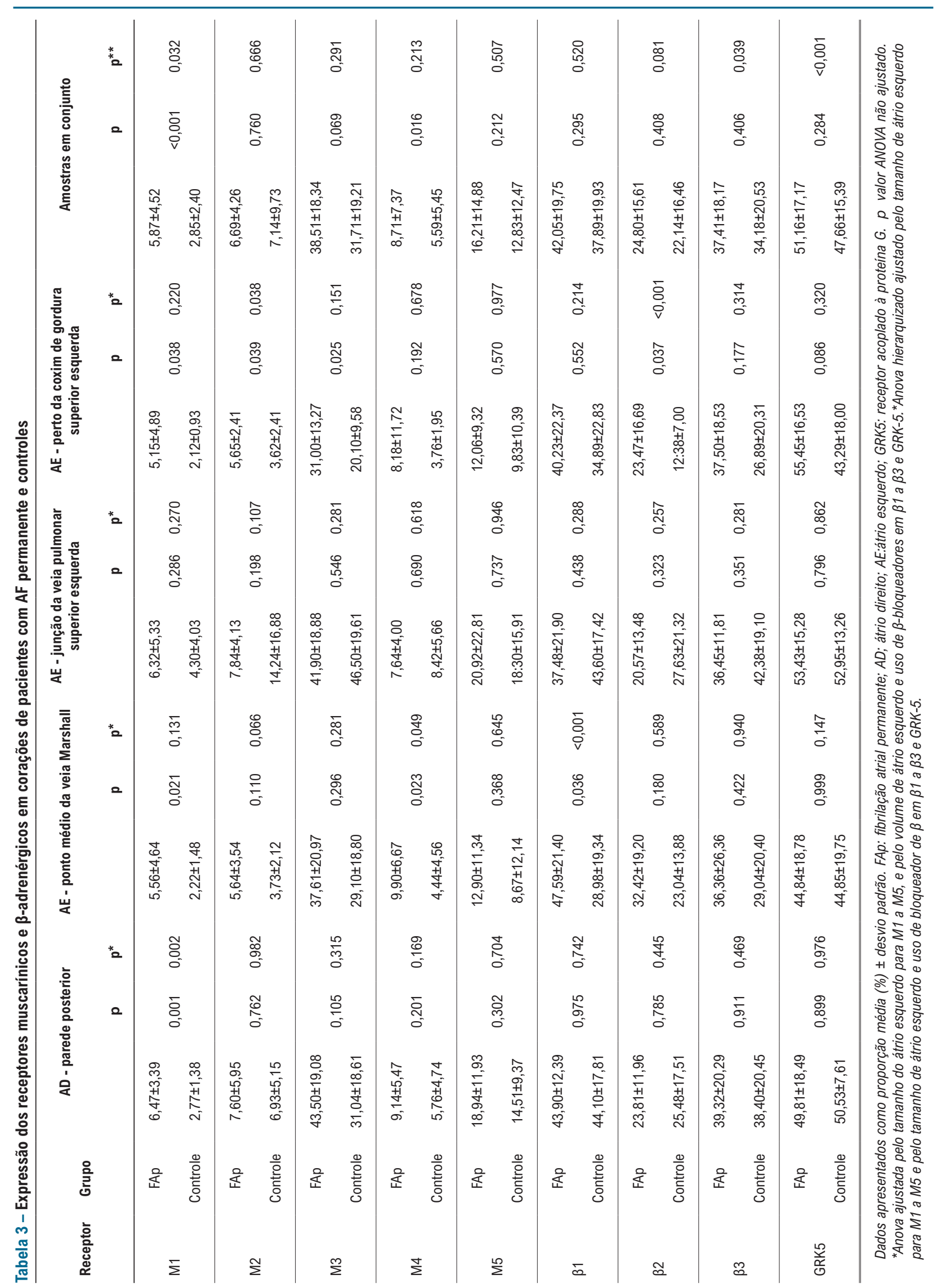


que as alterações que encontramos sejam não a causa, mas o efeito da FA, por mecanismo não esclarecido. Por outro lado, o desequilíbrio do SNACI, como demonstrado em estudos experimentais e eletrofisiológicos, pode ser causado por baixa atividade da inervação cardíaca autonômica (na qual a redução da área média dos nervos com manutenção geral da densidade de fibras poderia ter uma função, ainda que seja importante mencionar que não houve alteração na área de nervos), com aumento desproporcional da inervação simpática. É importante salientar que o aumento da expressão cardíaca de receptores muscarínicos, especialmente dos relacionados à atividade induzida por catecolaminas (M1, M2 e M4) e em regiões específicas relacionadas à FA (M1 e M3), aponta para a existência de possível desequilíbrio na atividade autonômica que poderia perpetuar essa arritmia de modo permanente em corações humanos, ao aumentar a sensibilidade a estímulos atriais causados pela acetilcolina.

Receptores $\beta$-adrenérgicos na fibrilação atrial permanente e o uso de $\beta$-bloqueadores

Em que pese a grande importância do controle $\beta$-adrenérgico do ritmo cardíaco, nossos dados indicam que não há diferença na expressão de seus receptores ou da quinase GRK-5 com o uso de $\beta$-bloqueadores.

Não foi encontrada diferença significativa nos receptores $\beta$-adrenérgicos tipos 1 ou 2 . Por outro lado, os receptores $\beta 3$ e GRK-5 estavam bastante aumentados nas amostras de pacientes com FA permanente.

\section{Considerações metodológicas e limitações do estudo}

Há relativamente poucos trabalhos que usam métodos anatomopatológicos para estudar arritmias cardíacas, principalmente porque grande parte das alterações subjacentes a elas são essencialmente eletrofisiológicas, com poucas repercussões morfológicas, e porque frequentemente requerem trabalhosos mapeamentos cardíacos. Porém, uma vez que tais desafios sejam encarados, esses métodos têm potencial de contribuir de forma significativa para o entendimento dessas doenças. Nossa abordagem no presente estudo foi a de verificar tipos e áreas das fibras nervosas autonômicas, a expressão de receptores muscarínicos e adrenérgicos e a quinase desses últimos (GRK-5) na fibrilação atrial humana.

Nossos achados demonstram que esse método é útil para identificar alterações eventualmente presentes (como nos receptores). Claramente, uma das limitações desse tipo de estudo é que a expressão morfológica das fibras nervosas e dos receptores não implica diretamente que sejam funcionais, mas pode-se inferir que mudanças em suas concentrações miocárdicas podem refletir alterações em sua atividade.

Vale reforçar a importância da escolha de controles adequados para os estudos patológicos: ainda que a FA em geral ocorra em pacientes como acompanhante de alguma doença estrutural, a maioria dos artigos anteriores utilizou corações normais como controles. ${ }^{11}$ Desse modo, é impossível determinar com precisão suficiente quais achados são, de fato, ligados à arritmia. Para evitar esse viés, nossos pacientes-controle tinham as mesmas doenças que aqueles com FA, como se tivéssemos "excluído" as doenças acima e abaixo de uma linha de fração, deixando apenas a arritmia para explicar as diferenças. Além disso, usamos amostras de pacientes com pelo menos 2 anos desde o diagnóstico, para ter certeza de que qualquer alteração potencial fosse fixa.

\section{Conclusões}

O aumento da inervação do sistema nervoso autônomo cardíaco intrínseco, assim como o remodelamento da expressão de receptores em regiões propensas a desencadear fibrilação atrial, podem ter uma função na condição de pacientes com fibrilação atrial permanente secundária à doença cardíaca estrutural.

\section{Agradecimentos}

Os autores agradecem a Solange Consorti e Ângela Santos por seu suporte técnico.

\section{Contribuição dos autores}

Concepção e desenho da pesquisa: Oliveira IM, Silva Júnior EL, Scanavacca MI, Gutierrez PS; Obtenção de dados: Oliveira IM, Silva Júnior EL, Martins YO; Análise e interpretação dos dados e Obtenção de financiamento: Oliveira IM, Silva Júnior EL, Gutierrez PS; Análise estatística: Oliveira IM, Rocha HAL; Redação do manuscrito: Oliveira IM, Silva Júnior EL, Martins YO, Rocha HAL, Scanavacca MI, Gutierrez PS; Revisão crítica do manuscrito quanto ao conteúdo intelectual importante: Oliveira IM, Martins YO, Scanavacca MI, Gutierrez PS.

\section{Potencial conflito de interesse}

Declaro não haver conflito de interesses pertinentes.

\section{Fontes de financiamento}

O presente estudo foi financiado pela FAPESP, processo no 07/56648-8.

\section{Vinculação acadêmica}

Este artigo é parte de tese de doutorado de Ítalo Martins de Oliveira, no Departamento de Cardiologia da Faculdade de Medicina da Universidade de São Paulo, e de Evilásio Leobino da Silva Júnior, pela mesma Faculdade, em conjunto com a Universidade Estadual do Ceará.

\section{Aprovação ética e consentimento informado}

Este estudo foi aprovado pelo Comitê de Ética do Instituto do Coração sob o número de protocolo 3043/07/118. Todos os procedimentos envolvidos nesse estudo estão de acordo com a Declaração de Helsinki de 1975, atualizada em 2013. 


\section{Referências}

1. Wann LS, Curtis AB, Ellenbogen KA, Estes NA, Ezekowitz MD, Jackman WM, et al. Management of patients with atrial fibrillation (compilation of 2006 ACCF/AHA/ESC and 2011 ACCF/AHA/HRS recommendations): a report of the American College of Cardiology/American Heart Association Task Force on practice guidelines. Circulation. 2013;127(18):1916-26.

2. Boldt A, Wetzel U, Lauschke J, Weigl J, Gummert J, Hindricks G, et al. Fibrosis in left atrial tissue of patients with atrial fibrillation with and without underlying mitral valve disease. Heart. 2004;90(4):400-5.

3. Oliveira IM, Oliveira BD, Scanavacca MI, Gutierrez PS. Fibrosis, myocardial crossings, disconnections, abrupt turns, and epicardial reflections: Do they play an actual role in human permanent atrial fibrillation? A controlled necropsy study. Cardiovasc Pathol. 2013;22(1):65-9.

4. Kumagai K, Ogawa M, Noguchi H, Yasuda T, Nakashima H, Saku K. Electrophysiologic properties of pulmonary veins assessed using a multielectrode basket cateter. J Am Coll Cardiol. 2004;43(12):2281-9.

5. Calkins H, Hindricks G, Cappato R, Kim Y, Saad EB, Aguinaga L, et al. 2017 HRS/EHRA/ECAS/APHRS/SOLAECE expert consensus statement on cateter ans surgical ablation of atrial fibrillation: Executive summary. Europace. 2018;20(1):157-208.

6. Scanavacca MI, Pisani CF, Hachul D, Lara S, Hardy C, Darrieux F, et al. Selective atrial vagal denervation guided by evoked vagal reflex to treat patients with paroxysmal atrial fibrillation. Circulation. 2006;114(9):876-85.

7. Carnagarin R, Kiuchi MG, Ho JK, Matthews VB, Schlaich MP. Sympathetic nervous system activation and its modulation: role in atrial fibrillation. Front Neurosci. 2019 Jan 23;12:1058.

8. Linz D, Elliott AD, Hohl M, Malik V, Schotten U, Dobrev D, et al. Role of autonomic nervous system in atrial fibrillation. Int J Cardiol. 2019 Jul $15 ; 287: 181-8$.

9. Tomita T, Takei M, Saikawa Y, Hanaoka T, Uchikawa S, Tsutsui H, et al. Role of autonomic tone in the initiation and termination of paroxysmal atrial fibrillation in patients without structural heart disease. J Cardiovasc Electrophysiol. 2003;14(6):559-64.

10. Berg MP, Hassink RJ, Baljé-Volkers C, Crijns HJGM. Role of the autonomic nervous system in vagal atrial fibrillation. Heart. 2003;89(3):333-5.

11. Razavi M, Zhang S, Yang D, Sanders RA, Kar B, Delapasse S, et al. Effects of pulmonary vein ablation on regional atrial vagal innervation and vulnerability to atrial fibrillation in dogs. J Cardiovasc Electrophysiol. 2005;16(8):879-84.

12. Kim DT, Lai AC, Hwang C, Fan LT, Karagueuzian HS, Chen PS, et al. The ligament of Marshall: a structural analysis in human hearts with implications for atrial arrhythmias. J Am Coll Cardiol. 2000;36(4):1324-7.
13. Ulphani JS, Arora R, Cain JH, Villuendas R, Shen S, Gordon D, et al. The ligament of Marshall as a parasympathetic conduit. Am J Physiol Heart Circ Physiol. 2007;293(3):H1629-35

14. Makino M, Inoue S, Matsuyama TA, Ogawa G, Sakai T, Kobayashi Y, et al Diverse myocardial extension and autonomic innervation on ligament of Marshall in humans. J Cardiovasc Electrophysiol. 2006;17(6):594-9.

15. Aiello VD, Higuchi ML, Lopes EA, Lopes AAB, Barbero-Marcial M, Ebaid M. An immunohistochemical study of arterial lesions due to pulmonary hypertension in patients with congenital heart defects. Cardiol Young. 1994;4(1):37-43.

16. Hopkins DA, Macdonald SE, Murphy DA, Armour JA. Pathology of intrinsic cardiac neurons from ischemic human hearts. Anat Rec. $2000 ; 259(4): 424-36$

17. Sharifov OF, Fedorov VV, Beloshapko GG, Glukhov AV, Yushmanova AV Rosenshtraukh LV. Roles of adrenergic and cholinergic stimulation in spontaneous atrial fibrillation in dogs. J Am Coll Cardiol. 2004;43(3):483-90.

18. Po SS, Scherlag BJ, Yamanashi WS, Edwards J, Zhou J, Wu R, et al. Experimental model for paroxysmal atrial fibrillation arising at the pulmonary vein-atrial junctions. Heart Rhythm. 2006;3(2):201-8.

19. Lin WS, Prakash VS, Tai CT, Hsieh MH, Tsai CF, Yu WC, et al. Pulmonary vein morphology in patients with paroxysmal atrial fibrillation initiated by ectopic beats originating from the pulmonary veins: implications for catheter ablation. Circulation. 2000;101(11):1274-81

20. Tan AY, Chen PS, Chen LS, Fishbein MC. Autonomic nerves in pulmonary veins. Heart Rhythm. 2007;4(3 Suppl):S57-60.

21. Caulfield MP, Birdsall NJ. International Union of Pharmacology. XVII. Classification of muscarinic acetylcholine receptors. Pharmacol Rev. 1998;50(2):279-90

22. Wang H, Han H, Zhang L, Shi H, Schram G, Nattel S, et al. Expression of multiple subtypes of muscarinic receptors and cellular distribution in the human heart. Mol Pharmacol. 2001;59(5):1029-36.

23. Hardouin SN, Richmond KN, Zimmerman A, Hamilton SE, Feigl EO Nathanson NM. Altered cardiovascular responses in mice lacking the $\mathrm{M}(1)$ muscarinic acetylcholine receptor. J Pharmacol Exp Ther. 2002;301(1):129-37

24. Shi H, Wang H, Li D, Nattel S, Wang Z. Differential alterations of receptor densities of three muscarinic acetylcholine receptor subtypes and current densities of the corresponding $\mathrm{K}+$ channels in canine atria with atria fibrillation induced by experimental congestive heart failure. Cell Physiol Biochem. 2004;14(1-2):31-40. 